\title{
Physics on the Terascale
}

\author{
J. Engelen
}

\section{The Terascale}

At an energy of order $10^{12} \mathrm{eV}$, i.e. $1 \mathrm{TeV}$, the fundamental interactions between quarks, leptons and gauge bosons need an additional ingredient in order to preserve unitarity. This ingredient is naturally provided by the Brout-Englert-Higgs mechanism. This mechanism invokes a scalar field with a 'Mexican hat' type potential, in which the ground state of the vacuum occurs at a finite value of the field ('symmetry breaking'); the quantum of this field, the Higgs boson, provides a way of 'generating' massive gauge bosons whilst preserving renormalizability of the theory. The existence of the Higgs boson has not yet been demonstrated experimentally; in fact the elucidation of 'the Higgs sector' represents a huge experimental program - arguably including the 'next large collider' ILC-CLIC that only begins with the discovery of the (or 'a') Higgs boson.

Furthermore, access to the Terascale holds the promise of more discoveries. A theoretically attractive scenario, in which the electro-weak and strong interactions unify at a scale of $10^{15} \mathrm{GeV}$ ('Grand Unification'), requires an additional ingredient to enter into the renormalization group equations on a scale of $1 \mathrm{TeV}$. This could mark the scale on which 'supersymmetry' is revealed and a new world of supersymmetric partners of all known particles is discovered.

Finally, and increasingly speculatively, the Terascale might give us access to 'large' (of the order of $0.1 \mathrm{~mm}$ ) extra dimensions, only open to gravity: this would open up possibilities of the experimental study of quantum gravity well below the Planck scale $\left(10^{19} \mathrm{GeV}\right)$.

\section{An accelerator for the Terascale}

The Large Hadron Collider was approved by the CERN Council in December 1996. Initial ideas can be traced back to the late 1970's, but even in 1996 an extensive $\mathrm{R} \& \mathrm{D}$ programme still had to be completed before the feasibility (and the cost) of the LHC could be established. The nominal energy of $14 \mathrm{TeV}$, i.e. $7 \mathrm{TeV}$ per beam, required new superconducting magnet technology; the nominal luminosity of $10^{34} \mathrm{~cm}^{-2} \mathrm{~s}^{-1}$ required, among other things, a novel collimation system.

Moreover, experiments able to cope with the interaction rate at this energy needed new concepts in detector technology and on-line selection of poten- tially interesting events ('triggering'). This is the subject of a separate presentation at this Symposium, by Peter Jenni (spokesman of the ATLAS collaboration). In addition, the huge amounts of data of 15 PetaBytes per year required a new approach to computing: the now operational worldwide LHC computing GRID (wLCG), also serving as a platform for applications outside high energy physics.

The LHC dipole magnets have all now been successfully produced, and the last magnet was installed in the tunnel in March 2008. In total 1232 of these magnets and about 400 quadrupole magnets (and a large number of smaller, higher order correction magnets) form the LHC lattice. The LHC is a marvel of technology in all its facets, crowned by the superconducting dipole magnets. They are $15 \mathrm{~m}$ in length, and feature two coils (for the two beams) in one 'cold mass' (flux return yoke). The coils consist of niobiumtitanium cables (out of 7 micron filaments) and are operated at a temperature of $1.9 \mathrm{~K}$, cooled by superfluid helium.

The LHC was successfully put into operation on September 10, 2008. In the period September 10September 19, 2008 this very complex machine proved to have been extremely well designed: injecting, circulating and 'capturing' (by the RF acceleration system) the beam was achieved in a matter of days. Preparations for first collisions were in full swing when an unfortunate incident revealed a weakness in one of the joints between two superconducting cables between two magnets. There are more than 10000 such connections in the LHC, but one of them had a resistance of $\sim 100$ nano-ohm (instead of less than 1 , as in the specifications - a few additional 'suspect' joints were identified during subsequent inspections). The dissipated energy (at a current of $10 \mathrm{kA}$ ) led to warm up and eventual failure of the joint. The loose cable subsequently discharged, burning a hole in the pipe carrying the liquid helium. The helium escaping into the vacuum system caused a pressure wave that damaged (and in a number of cases dislodged) magnets over a distance of several hundred meters. The repairs and the implementation of additional diagnostics (and of some measures to limit the damage should such an event occur again, but it should not!) are estimated to take a full year, such that restart of the accelerator is foreseen for October 2009. (Note added: meanwhile the LHC has very successfully resumed operation in November 2009).

\footnotetext{
Brief summary of a colloquium given in honor of Professor J. Niederle on the occasion of his $70^{\text {th }}$ birthday
} 


\section{Conclusion}

Research into the interactions of elementary particles and fields is at the eve of a new era. The Large Hadron Collider, a marvel of technology and a wonderful example of European leadership in worldwide collaboration will allow the exploration of new and uncharted territory, where exciting new phenomena are waiting to be discovered.

\section{Finally}

Jiri Niederle has contributed prominently to the European leadership referred to in the previous paragraph: as the Czech delegate, he is a long-time member of the CERN Council; on the basis of his authority as a prominent scientist he has successfully helped to steer CERN in the right direction!

\section{References}

[1] The Large Hadron Collider: a Marvel of Technology. EPFL Press, 2009, edited by Lyndon Evans.

Jos Engelen

NIKHEF and NWO - the Netherlands Organisation for Scientific Research

$\mathrm{P} / \mathrm{O}$ BOX 93138

NL 2509 AC Den Haag, Netherlands 